\title{
LEVELS OF POSITIVE DEFINITE TERNARY QUADRATIC FORMS
}

\author{
J. LARRY LEHMAN
}

\begin{abstract}
The level $N$ and squarefree character $q$ of a positive definite ternary quadratic form are defined so that its associated modular form has level $N$ and character $\chi_{q}$. We define a collection of correspondences between classes of quadratic forms having the same level and different discriminants. This makes practical a method for finding representatives of all classes of ternary forms having a given level. We also give a formula for the number of genera of ternary forms with a given level and character.
\end{abstract}

\section{INTRODUCTION}

In this article, we consider some questions concerning the classification of positive definite ternary quadratic forms. Our motivation is the connection between quadratic forms and modular forms which is given in the theorem below. We first recall some notation and terminology concerning modular forms.

Define a symbol $(a / b)$ for $a, b \in \mathbb{Z}$ by the following conditions:

(1) $(a / b)$ is the Legendre symbol if $b$ is an odd prime.

(2) $(a / 2)=(-1)^{\left(a^{2}-1\right) / 8}$ if $a$ is odd.

(3) $(a /-1)=1$ if $a \geq 0,(a /-1)=-1$ if $a<0$.

(4) $(a / b)=0$ if $\operatorname{gcd}(a, b)>1,(1 / 0)=1,(a / 0)=0$ if $a \neq 1$.

(5) $(a / b c)=(a / b) \cdot(a / c)$ for all $b, c \in \mathbb{Z}$.

If $t$ is a nonzero integer, define a function $\chi_{t}$ on the integers as follows: Let $t=q r^{2}$ with $q$ squarefree. If $q \equiv 1(\bmod 4)$, let $D=q$. If $q \equiv 2,3$ $(\bmod 4)$, let $D=4 q$. Then $\chi_{t}(n)=(D / n)$ for all $n \in \mathbb{Z}$. The function $\chi_{t}$ is a quadratic Dirichlet character with conductor $|D|[11]$.

Let $k$ be an integer, $N$ a positive integer (divisible by 4 if $k$ is odd), and $\chi$ a character modulo $N$. Let $\Gamma_{0}(N)$ be the subgroup of $\mathrm{SL}_{2}(\mathbb{Z})$ consisting of all $\left[\begin{array}{ll}a & b \\ c & d\end{array}\right]$ with $c \equiv 0(\bmod N)$. A modular form $\theta$ is said to have weight $k / 2$, level $N$, and character $\chi$ if for all $\gamma=\left[\begin{array}{ll}a & b \\ c & d\end{array}\right] \in \Gamma_{0}(N)$ and all $z \in \mathbb{C}$ with $\operatorname{Im}(z)>0$,

$$
\theta\left(\frac{a z+b}{c z+d}\right)= \begin{cases}\chi(d) \cdot(c z+d)^{k / 2} \cdot \theta(z) & \text { if } k \text { is even, } \\ \chi(d) \cdot j(\gamma, z)^{k} \cdot \theta(z) & \text { if } k \text { is odd. }\end{cases}
$$

Here, $j(\gamma, z)=\varepsilon_{d}^{-1} \chi_{c}(d)(c z+d)^{1 / 2}$, where $\varepsilon_{d}=1$ or $i$ as $d \equiv 1$ or 3 $(\bmod 4)$. Denote the vector space of all such modular forms as $M_{k / 2}(N, \chi)$,

Received July 24, 1990; revised February 25, 1991.

1991 Mathematics Subject Classification. Primary 11E20; Secondary 11E45. 
and its subspace of cusp forms as $S_{k / 2}(N, \chi)$. (See [12] or [7] for more background on modular forms, particularly those of half-integral weight.)

Theorem (Shimura [12]). Let $f\left(x_{1}, \ldots, x_{n}\right)$ be a positive definite quadratic form having integer coefficients. Let $A$ be the $n \times n$ matrix

$$
A=\left[\frac{\partial^{2} f}{\partial x_{i} \partial x_{j}}\right] \text {. }
$$

Define $N$ to be the smallest positive integer so that $N A^{-1}$ is an even matrix, that is, has integral entries, and even integers on the main diagonal. Let $\theta(f)=\theta_{f}(z)$ be defined by

$$
\theta_{f}(z)=\sum q^{f\left(m_{1}, \ldots, m_{n}\right)}
$$

where $q=e^{2 \pi i z}$, and the sum is taken over all $n$-tuples $\left(m_{1}, \ldots, m_{n}\right)$ in $\mathbb{Z}^{n}$. Then $\theta(f) \in M_{n / 2}\left(N, \chi_{d}\right)$, where $d=\operatorname{det}(A)$ if $n \equiv 0(\bmod 4), d=-\operatorname{det}(A)$ if $n \equiv 2(\bmod 4)$, and $d=\operatorname{det}(A) / 2$ if $n$ is odd.

Remarks. This theorem is a special case of Proposition 2.1 in [12]. Shimura's proposition generalizes results of Hecke and Schoeneberg in the case when $n$ is even, and of Pfetzer when $n$ is odd (see [12] for references). It is not hard to see that $\operatorname{det}(A)$ is even if $n$ is odd, so the discriminant $d$ of $f$ is an integer in each case. In saying that $\chi_{d}$ is the character of $\theta(f)$, we mean that $\theta(f)$ has character $\chi$ such that $\chi(a)=\chi_{d}(a)$ if $\operatorname{gcd}(a, N)=1$. (By definition, $\chi(a)=0$ if $\operatorname{gcd}(a, N)>1$.) Suppose that $g=c f$, with $c$ a positive integer. Then $\theta(g)$ has weight $n / 2$ and level $c N$. Its character is $\chi_{d}$ if $n$ is even, $\chi_{c d}$ if $n$ is odd. As a power series in $q, \theta(g)$ is the same as $\theta(f)$ with all exponents multiplied by $c$. So we can restrict our attention to the case where $f$ is primitive, that is, where the greatest common divisor of the coefficients of $f$ is 1 . Finally, we have that if $f_{1}$ and $f_{2}$ are in the same genus of forms (see $\S 3)$, then $\theta\left(f_{1}\right)-\theta\left(f_{2}\right) \in S_{n / 2}\left(N, \chi_{d}\right)$ [10].

Attempts have been made to use quadratic forms to describe a space of modular or cusp forms of a given weight, level, and character. In formal terms, this can be considered as a special case of the "basis problem," which was successfully dealt with in [5] in the case in which the weight is an integer $k \geq 2$. Serre and Stark [11] found bases for all spaces of forms of weight $1 / 2$, using theta series, which may be defined in terms of quadratic forms. In [8], the author employed quadratic forms to construct a basis for $S_{3 / 2}\left(196, \chi_{7}\right)$, in order to fully compute the effects of the Hecke operators on this space, and the Shimura correspondence on associated eigenforms. Obviously, it would be helpful in this application to be able to find all primitive quadratic forms which lead to a particular value of the level $N$. If $n=1$, then this is trivial, as there is only one primitive form in that case. In the case of binary forms $(n=2)$, this problem is the same as that of finding all primitive forms of a given discriminant. For if $f\left(x_{1}, x_{2}\right)=a_{11} x_{1}^{2}+a_{12} x_{1} x_{2}+a_{22} x_{2}^{2}$ is primitive, then

$$
A=\left[\begin{array}{cc}
2 a_{11} & a_{12} \\
a_{12} & 2 a_{22}
\end{array}\right] \text { and } A^{-1}=\frac{1}{\operatorname{det}(A)}\left[\begin{array}{cc}
2 a_{22} & -a_{12} \\
-a_{12} & 2 a_{11}
\end{array}\right]
$$

leading to the conclusion that $N=\operatorname{det}(A)$ in every case.

When we look at ternary forms $(n=3)$, however, this is no longer the case. For example, let

$$
f\left(x_{1}, x_{2}, x_{3}\right)=x_{1}^{2}+2 x_{2}^{2}+8 x_{3}^{2}
$$


and

$$
g\left(x_{1}, x_{2}, x_{3}\right)=3 x_{1}^{2}+11 x_{2}^{2}+11 x_{3}^{2}-10 x_{2} x_{3}-2 x_{1} x_{3}-2 x_{1} x_{2} .
$$

Then $\theta(f)$ and $\theta(g)$ are both weight $3 / 2$ forms of level 32 and trivial character. But $f$ has discriminant 64 while that of $g$ is 1024 .

This example illustrates another point. Extensive tables of positive definite ternary quadratic forms, grouped by discriminant, have been compiled. In particular, the tables of Brandt and Intrau [1] list (in over 200 pages) all reduced ternary forms with $d \leq 1000$. But, as we see above, a modular form of relatively small level may arise from a quadratic form with a large discriminant.

In this article, we will consider the following question: Is it possible to find all primitive, positive definite, ternary quadratic forms whose associated modular forms possess a particular level? We will show that this is possible in general, and illustrate a practical method for doing so for a large number of values of the level.

\section{TERNARY QUADRATIC FORMS}

The literature on quadratic forms is extensive and highly developed. We will take an elementary approach to the subject, focusing narrowly on positive definite ternary quadratic forms which are defined over the integers. However, our approach is unique in that it stresses the level throughout as the invariant of importance for a quadratic form.

Let $f$ be a ternary quadratic form with integer coefficients, given by the equation

$$
f(x, y, z)=a x^{2}+b y^{2}+c z^{2}+r y z+s x z+t x y .
$$

Unless otherwise stated, we assume that $f$ is positive definite (that is, that $f(x, y, z)>0$ for real numbers $x, y, z$ unless $x=y=z=0$ ) and primitive $(\operatorname{gcd}(a, b, c, r, s, t)=1)$. (Note that we do not follow the "classically integral" definition, which requires that $r, s$, and $t$ be even integers. Some results quoted below, particularly those of Dickson [4], have been restated to account for this difference in definitions.) We will also denote $f$ by the array $f=\left(\begin{array}{lll}a & b & c \\ r & s & t\end{array}\right)$.

Define the matrix of $f$ to be

$$
A=A_{f}=\left[\begin{array}{ccc}
2 a & t & s \\
t & 2 b & r \\
s & r & 2 c
\end{array}\right]
$$

We will say that a $3 \times 3$ matrix is primitive if it is the matrix of a primitive ternary form. Define the discriminant of $f$ to be

$$
d=d_{f}=\frac{\operatorname{det}(A)}{2}=4 a b c+r s t-a r^{2}-b s^{2}-c t^{2} \text {. }
$$

Let $A_{i j}$ be the $i, j$-cofactor of $A$. That is,

$$
\begin{array}{ll}
A_{11}=4 b c-r^{2}, & A_{23}=s t-2 a r=A_{32}, \\
A_{22}=4 a c-s^{2}, & A_{13}=r t-2 b s=A_{31}, \\
A_{33}=4 a b-t^{2}, & A_{12}=r s-2 c t=A_{21} .
\end{array}
$$


Define the divisor of $f$ to be the positive integer

$$
m=m_{f}=\operatorname{gcd}\left(A_{11}, A_{22}, A_{33}, 2 A_{23}, 2 A_{13}, 2 A_{12}\right) \text {. }
$$

Let $\alpha=A_{11} / m, \beta=A_{22} / m, \gamma=A_{33} / m, \rho=2 A_{23} / m, \sigma=2 A_{13} / m$, and $\tau=2 A_{12} / \mathrm{m}$. Define the reciprocal of $f$ to be the ternary form

$$
\phi(x, y, z)=\alpha x^{2}+\beta y^{2}+\gamma z^{2}+\rho y z+\sigma x z+\tau x y .
$$

It is clear that $\phi$ is a primitive positive definite form.

The matrix of $\phi$ is

$$
A_{\phi}=\left[\begin{array}{ccc}
2 \alpha & \tau & \sigma \\
\tau & 2 \beta & \rho \\
\sigma & \rho & 2 \gamma
\end{array}\right]=\frac{2}{m}\left[\begin{array}{ccc}
A_{11} & A_{21} & A_{31} \\
A_{12} & A_{22} & A_{32} \\
A_{13} & A_{23} & A_{33}
\end{array}\right]=\frac{2 \operatorname{det}(A)}{m} A_{f}^{-1}
$$

by the usual cofactor results. So $A_{\phi}=\frac{4 d}{m} A_{f}^{-1}$. Notice that $m$ divides $4 d$ because

$$
4 d=2 \operatorname{det}(A)=4 a\left(A_{11}\right)+t\left(2 A_{12}\right)+s\left(2 A_{13}\right) .
$$

Define the level of $f$ to be the positive integer $N=N_{f}=4 d_{f} / m_{f}$. Note that, as in the introduction, $N$ is the smallest positive integer such that $N A_{f}^{-1}$ is even. We can also describe the level of $f$ as the unique positive integer $N$ so that $N A_{f}^{-1}$ is a primitive matrix.

Now consider the definitions above applied to the primitive form $\phi$. Since $A_{\phi}=N_{f} A_{f}^{-1}$, the discriminant of $\phi$ is

$$
d_{\phi}=\frac{\operatorname{det}\left(A_{\phi}\right)}{2}=\frac{N_{f}^{3}}{2} \operatorname{det}\left(A_{f}^{-1}\right)=\frac{N_{f}^{3}}{4 d_{f}} .
$$

Let $m_{\phi}$ be the divisor of $\phi$, and let $N_{\phi}=4 d_{\phi} / m_{\phi}$ be its level. Let $F$ be the reciprocal of $\phi$. Then

$$
A_{F}=N_{\phi} A_{\phi}^{-1}=N_{\phi}\left(N_{f} A_{f}^{-1}\right)^{-1}=\frac{N_{\phi}}{N_{f}} A_{f} .
$$

But $F$ is a primitive form by the definition of the reciprocal. So $A_{F}$ is a primitive matrix, as is $A_{f}$. Clearly, the only way in which a positive scalar multiple of a primitive matrix can be primitive is if the scalar is 1 . Therefore, $f$ is the reciprocal of $\phi$. Furthermore, we have the following important fact.

Theorem 1. Let $f$ be a primitive, positive definite, ternary quadratic form, and let $\phi$ be its reciprocal. Then $f$ and $\phi$ have the same level.

Fix the following notation now. Considered as constants depending on $f$, denote $d_{f}$ by $d, m_{f}$ by $m, d_{\phi}$ by $\delta, m_{\phi}$ by $\mu$, and the common value of $N_{f}$ and $N_{\phi}$ by $N$. Each of these quantities is a positive integer.

Ternary forms $f$ and $g$ are said to be equivalent, $f \sim g$, if there is a unimodular matrix $U=\left[u_{i j}\right]$ so that $A_{g}=U A_{f} U^{t}$. (That is, $U$ has integer entries and $\operatorname{det}(U)= \pm 1 ; U^{t}$ is its transpose.) In this case, the coefficients of $g$ can be expressed explicitly in terms of $f$ as follows. For $i=1,2,3$, let $\mathbf{u}_{i}=\left(u_{i 1}, u_{i 2}, u_{i 3}\right)$. Suppose that $g\left(x_{1}, x_{2}, x_{3}\right)=\sum_{i \leq j} a_{i j} x_{i} x_{j}$. Then

$$
a_{i j}= \begin{cases}f\left(\mathbf{u}_{i}\right) & \text { if } i=j \\ f\left(\mathbf{u}_{i}+\mathbf{u}_{j}\right)-f\left(\mathbf{u}_{i}\right)-f\left(\mathbf{u}_{j}\right) & \text { if } i \neq j .\end{cases}
$$

Equivalent forms are said to belong to the same class. Clearly, if $f \sim g$, then $d_{f}=d_{g}$. 
Proposition 1. The level of a form is a class invariant. That is, if $f \sim g$, then $N_{f}=N_{g}$.

Proof. If some prime divides each coefficient of $f$, then by equation (3) it divides each coefficient of $g$. It follows that $f$ is primitive if and only if $g$ is primitive. Now $N_{f} A_{g}^{-1}=V A_{\phi} V^{t}$, where $V=\left(U^{t}\right)^{-1}$ is unimodular. Since $\phi$ is a primitive form, $N_{f} A_{g}^{-1}$ must be a primitive matrix. But $N_{g}$ is the unique positive integer so that $N_{g} A_{g}^{-1}$ is primitive. Therefore $N_{f}=N_{g}$.

Corollary 1. If $f$ and $g$ are equivalent, then their reciprocals are equivalent as well.

From the equations $\delta=N^{3} / 4 d, m=4 d / N$, and $\mu=4 \delta / N=N^{2} / d$, we see that $m, \mu$, and $\delta$ are also class invariants. Notice also that $m \mu=4 N$, $m^{2} \mu=16 d$, and $m \mu^{2}=16 \delta$. From these latter two equations we can see that if $m$ is odd, then $16 \mid \mu$, and if $\mu$ is odd, then $16 \mid m$. But $m$ is odd if and only if one of $A_{11}, A_{22}, A_{33}$ is odd. This is the case if and only if one of $r$, $s, t$ is odd. If not, then it is easy to see that $4 \mid \mathrm{m}$. Similarly, either $\mu$ is odd or $4 \mid \mu$. In any case, we see that $16 \mid m \mu$ and thus that $4 \mid N$.

Now suppose that $f$ is a ternary form having a given level $N$. What can be concluded about the discriminant $d$ of such a form? First note that if $p \mid d$, then $p \mid \mu d$, so $p \mid N^{2}$ and $p \mid N$. So $d$ cannot be divisible by any prime which does not divide $N$. Let $p$ be an odd prime and suppose that $p^{g} \| N$ (that is, $N$ is divisible by $p^{g}$, but not by $p^{g+1}$ ). Suppose that $p^{h} \| d$. From the fact that $m=4 d / N$ and $\mu=N^{2} / d$ are integers, we see that $g \leq h \leq 2 g$. Suppose that $2^{g} \| N$ (so that $g \geq 2$ ) and that $2^{h} \| d$. We can now conclude that $h+2 \geq g$ and $2 g \geq h$, that is, $g-2 \leq h \leq 2 g$. But as noted in the previous paragraph, $m$ is either odd or divisible by 4 , and likewise for $\mu$. So we see that $h \neq g-1$ and $h \neq 2 g-1$ in this case. (If $f$ is a quadratic form in an even number of variables, then it is known that $N$ and $d$ are divisible by the same prime factors [9]. Note that for ternary forms, we may have $d$ odd although $N$ is even.)

There is an additional restriction on discriminant values. First note the following result which we will use on several occasions.

Proposition 2 [4, pp. 12-17]. Let $f$ be a ternary form. Let $m$ be its divisor and $\mu$ be the divisor of its reciprocal. Then $f$ is equivalent to a form $\left(\begin{array}{lll}a & b & c \\ r & s & t\end{array}\right)$, having reciprocal $\left(\begin{array}{lll}\alpha & \beta & \gamma \\ \rho & \sigma & \tau\end{array}\right)$, so that $a$ and $\gamma$ are relatively prime to each other and to $m \mu$.

Lemma 1. There is no primitive ternary form $f$ with divisor $m$, whose reciprocal has divisor $\mu$, so that $m$ and $\mu$ are both squares and either $m$ or $\mu$ is odd.

Proof. Suppose that $f$ is such a form. We may assume that $f$ and its reciprocal are as given in Proposition 2. In particular then, $a$ and $\gamma$ are odd and positive, and so we may consider the Jacobi symbols $(m \gamma / a)$ and $(\mu a / \gamma)$. By the definition of the reciprocal, we have that $m \gamma=4 a b-t^{2}$ and $\mu a=4 \beta \gamma-\rho^{2}$. Since $m$ and $\mu$ are squares, it follows that

$$
\left(\frac{\gamma}{a}\right)=\left(\frac{m \gamma}{a}\right)=\left(\frac{4 a b-t^{2}}{a}\right)=\left(\frac{-1}{a}\right)
$$


and

$$
\left(\frac{a}{\gamma}\right)=\left(\frac{\mu a}{\gamma}\right)=\left(\frac{4 \beta \gamma-\rho^{2}}{\gamma}\right)=\left(\frac{-1}{\gamma}\right) .
$$

So

$$
\left(\frac{\gamma}{a}\right)\left(\frac{a}{\gamma}\right)=\left(\frac{-1}{a}\right)\left(\frac{-1}{\gamma}\right),
$$

and by Quadratic Reciprocity,

$$
(-1)^{(a-1)(\gamma-1) / 4}=(-1)^{(a-1) / 2}(-1)^{(\gamma-1) / 2} .
$$

But if $m$ is odd (and a square), then $\gamma \equiv m \gamma \equiv 4 a b-t^{2} \equiv-1(\bmod 4)$. Then it follows that

$$
(-1)^{(a-1) / 2}=-(-1)^{(a-1) / 2},
$$

which is impossible. There is a similar contradiction if $\mu$ is odd. So $f$ cannot exist under these conditions.

The divisors $m$ and $\mu$ are both squares if and only if $N=m \mu / 4$ and $d=m N / 4$ are both squares. We summarize the above results as:

Theorem 2. Let $f$ be a primitive, positive definite, ternary quadratic form with level $N$ and discriminant $d$. Suppose that

$$
N=2^{n_{0}} p_{1}^{n_{1}} \ldots p_{k}^{n_{k}}
$$

is the prime factorization of $N$. Then $n_{0} \geq 2$ and $d$ is of the form

$$
d=2^{d_{0}} p_{1}^{d_{1}} \ldots p_{k}^{d_{k}}
$$

with the following restrictions on exponents:

(1) $d_{0}=n_{0}-2, d_{0}=2 n_{0}$, or $n_{0} \leq d_{0} \leq 2 n_{0}-2$, and

(2) for $1 \leq i \leq k, n_{i} \leq d_{i} \leq 2 n_{i}$.

Furthermore, if $n_{i}$ is even for $0 \leq i \leq k$, then either $n_{0} \leq d_{0} \leq 2 n_{0}-2$, or $d_{i}$ is odd for some $1 \leq i \leq k$.

In particular, we see that given a value $N$, there is only a finite number of values $d$ so that a ternary form could have level $N$ and discriminant $d$. These values are explicitly calculable in terms of $N$. (In the following sections, when we write that $N$ and $d$ are given by equations (4) and (5), we will assume that they satisfy the conditions on exponents which are given in Theorem 2 . In $\S 3$, we will see that there is in fact a ternary form for every level $N$ and discriminant $d$ which are allowed by this theorem.)

\section{CONSTRUCTION OF ALL FORMS OF A GIVEN LEVEL}

Given a value $d$, it is possible (in theory) to find a representative of each class of primitive, positive definite, ternary quadratic forms having discriminant $d$. We sketch the method here.

Proposition 3 [4, pp. 155-179]. Let $f$ be a ternary form given by equation (1). Say that $f$ is reduced if the following are true:

(1) $a \leq b \leq c$;

(2) $r, s$, and $t$ are all positive or all nonpositive;

(3) $a \geq|t| ; \quad a \geq|s| ; \quad b \geq|r|$; 
(4) $a+b+r+s+t \geq 0$;

(5) $a=t \Rightarrow s \leq 2 r ; a=s \Rightarrow t \leq 2 r ; b=r \Rightarrow t \leq 2 s$;

(6) $a=-t \Rightarrow s=0 ; a=-s \Rightarrow t=0 ; \quad b=-r \Rightarrow t=0$;

(7) $a+b+r+s+t=0 \Rightarrow 2 a+2 s+t \leq 0$;

(8) $a=b \Rightarrow|r| \leq|s| ; \quad b=c \Rightarrow|s| \leq|t|$.

Then every primitive, positive definite, ternary form is equivalent to one and only one reduced form. Also, if $f$ is reduced and has discriminant $d$, then $d / 4 \leq a b c \leq d / 2$.

Remark. The above criteria for a reduced form were first provided by Eisenstein. Other definitions are possible. 'In particular, a form which is reduced by this definition is not necessarily "Minkowski reduced," a definition which requires in part that $r+s+t \leq a+b$ in all cases [3, p. 396].

This means that there is only a finite number of possibilities for the coefficients of a reduced form having a given discriminant. In particular, if $f$ is a reduced form, with discriminant $d$, given by equation (1), then

$$
1 \leq a \leq \sqrt[3]{d / 2}, \quad a \leq b \leq \sqrt{d / 2 a}, \quad \max (b, d / 4 a b) \leq c \leq d / 2 a b,
$$

and either

$$
-b \leq r \leq 0, \quad-a \leq s \leq 0, \quad-a \leq t \leq 0
$$

or

$$
1 \leq r \leq b, \quad 1 \leq s \leq a, \quad 1 \leq t \leq a .
$$

Starting with a given value of $N$, we could use Theorem 2 to find the finite collection of potential discriminants for forms of level $N$. For each such discriminant $d$, the finite collection of possible coefficients could be tested. Thus, it is theoretically possible to find all reduced forms of a given level. Of course, the larger $d$ is, the more potential coefficients have to be tested. Since for a given $N$, a corresponding $d$ might be as large as $N^{2}$, this direct method could become unworkable for a relatively small value of the level. However, we will note several results which allow us to restrict this search process, thus making it much more practical.

First note that, in some cases, we can place additional restrictions on the potential coefficients of forms, owing to the fact that we want only forms of a specific level. Suppose that $f$, given by equation (1), is a reduced form of level $N$ and discriminant $d$, having reciprocal $\phi$ as in equation (2). Let $m=4 d / N$ and $\mu=N^{2} / d$ be the respective divisors of $f$ and $\phi$. Then:

(1) If $m$ is even, then $r, s$, and $t$ must also be even. This is because $m$ is the greatest common divisor of a collection of integers including $4 b c-r^{2}$, $4 a c-s^{2}$, and $4 a b-t^{2}$.

(2) If $\mu$ is odd, then there are restrictions on the coefficients $a, b$, and $c$. We know that $\mu a=4 \beta \gamma-\rho^{2}$. If $a$ is even, then $\rho$ is even, and so $4 \mid \mu a$ and $4 \mid a$. If $a$ is odd, then $\rho$ is odd, and $\mu a \equiv-\rho^{2} \equiv-1(\bmod 4)$. So either $a \equiv 0$ or $a \equiv-\mu(\bmod 4)$. The same is true with $b$ or $c$ in place of $a$.

(3) Since $m \leq m \gamma=4 a b-t^{2} \leq 4 a b$, we have that $b \geq m / 4 a$. (It is worth noting that then we have $c \leq d / 2 a b \leq 2 d / m=N / 2$. Thus $N / 2$ is an upper limit for the absolute values of the coefficients of a reduced form of level $N$, independent of its discriminant.) 
More importantly though, we may use a collection of functions between classes of forms having a given level to cut down on the number of discriminants for which this coefficient-testing process must be carried out. Let $C(N, d)$ denote the set of all classes of positive definite ternary forms having level $N$ and discriminant $d$. If $f$ is a ternary form, let $\bar{f}$ denote the class to which $f$ belongs. Our next theorem restates some earlier results (Theorem 1 and Corollary 1).

Theorem 3. There is a one-to-one correspondence between the sets $C(N, d)$ and $C(N, \delta)$, where $\delta=N^{3} / 4 d$. This correspondence is provided by the mapping $\bar{f} \mapsto \bar{\phi}$, where $\phi$ is the reciprocal of $f$.

Since $d$ and $\delta$ are inversely related, we can immediately eliminate the discriminants associated with $N$ for which $d>\frac{1}{2} \sqrt{N^{3}}$. The following result allows us to restrict our attention further.

Theorem 4. Let $N$ and $d$ be given by equations (4) and (5). Suppose that $p^{g} \| N$ and $p^{h} \| d$ for some odd prime $p$. Write $d$ as $p^{h} d^{\prime}$. Then there is a one-to-one correspondence between $C\left(N, p^{h} d^{\prime}\right)$ and $C\left(N, p^{3 g-h} d^{\prime}\right)$.

Before we describe this correspondence, we need the following lemma.

Lemma 2. Let $f$ be a positive definite, primitive ternary form with level $N$ and divisor $m$. Suppose that $p^{i} \| N$ and $p^{j} \| m$ for some odd prime $p$ and positive

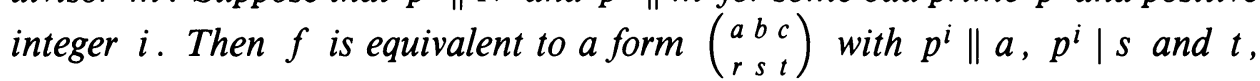
$p^{j} \mid b$ and $r$, and $p \nmid c$. If $0<j<i$, then we can assume that $p^{j} \| b$.

Proof. By Proposition 2, we may assume at the start that $f$ has reciprocal $\left(\begin{array}{lll}\alpha & \beta & \gamma \\ \rho & \sigma & \tau\end{array}\right)$ with $\gamma$ not divisible by $p$. Let $g=\operatorname{gcd}(\sigma, \rho, 2 \gamma)$, so that $p \nmid g$. We can form a unimodular matrix $U$ whose first row is $\left[\begin{array}{lll}\sigma / g & \rho / g & 2 \gamma / g\end{array}\right]$. Let $A$ be the matrix of $f$. Then the first row of $U A$ is $\left[\begin{array}{lll}0 & 0 & N / g\end{array}\right]$. (This can be seen from the fact that the third row of $A^{-1}$ is $\left[\begin{array}{lll}\sigma / N & \rho / N & 2 \gamma / N\end{array}\right]$.) So then, if $U=\left[u_{i j}\right]$, the first row of $U A U^{t}$ is $\left[\begin{array}{lll}2 \gamma N / g^{2} & u_{23} N / g & u_{33} N / g\end{array}\right]$.

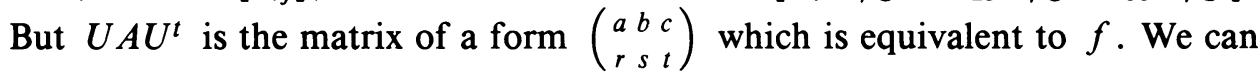
see that $a=\gamma N / g^{2}, s=u_{33} N / g$, and $t=u_{23} N / g$. Since $p^{i} \| N, p \nmid g$, and $p \nmid \gamma$, it follows that $p^{i} \| a$ and that $p^{i} \mid s, t$.

Since $f$ is primitive, one of the remaining coefficients must be relatively prime to $p$. It is easy to see that

$$
\left(\begin{array}{lll}
a & b & c \\
r & s & t
\end{array}\right) \sim\left(\begin{array}{lll}
a & c & b \\
r & t & s
\end{array}\right) \sim\left(\begin{array}{ccc}
a & b+c+r & c \\
2 c+r & s & s+t
\end{array}\right),
$$

so we may assume that either $b$ or $c$ is not divisible by $p$ without affecting the previous results concerning $a, s$, and $t$. If $j=0$, assume that $p \nmid c$. The proof is complete in that case.

If $j>0$, assume that $p \nmid b$. Let $g=\operatorname{gcd}(-r, 2 b)$, so that $p \nmid g$. We can form a unimodular matrix

$$
U=\left[\begin{array}{ccc}
1 & 0 & 0 \\
0 & -r / g & 2 b / g \\
0 & u & v
\end{array}\right]
$$




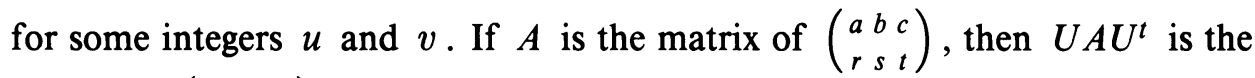

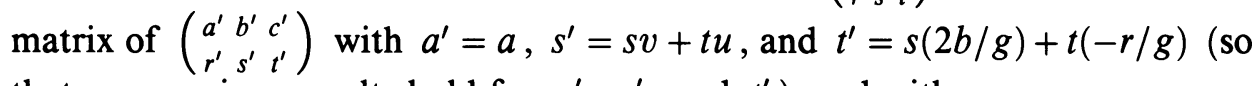
that our previous results hold for $a^{\prime}, s^{\prime}$, and $\left.t^{\prime}\right)$, and with

$$
b^{\prime}=b\left(4 b c-r^{2}\right) / g^{2} \text { and } r^{\prime}=v\left(4 b c-r^{2}\right) / g .
$$

The divisor $m$ is the greatest common divisor of a collection of integers which includes $4 b c-r^{2}$, and it is easy to see that each of the other integers is divisible by $p^{i}$. So we see that $p^{j} \mid b^{\prime}, r^{\prime}$, and if $j<i$, then $p^{j} \| b^{\prime}$. Since $f$ is primitive, $p$ cannot divide $c^{\prime}$. So the proof of Lemma 2 is complete.

Proof of Theorem 4. Let $\bar{f} \in C\left(N, p^{h} d^{\prime}\right)$. Since $m=4 d / N$, we may, by Lemma 2, assume that

$$
f=\left(\begin{array}{ccc}
p^{g} a & p^{h-g} b & c \\
p^{h-g_{r}} & p^{g} s & p^{g} t
\end{array}\right)
$$

with $a, b, c, r, s$, and $t$ integers, $p \nmid a c$. Let

$$
f_{p}=\left(\begin{array}{ccc}
a & p^{2 g-h} b & p^{g} c \\
p^{g} r & p^{g} s & p^{2 g-h} t
\end{array}\right) .
$$

Notice that $f_{p}$ is a primitive form. We will show that $\psi: C\left(N, p^{h} d^{\prime}\right) \rightarrow$ $C\left(N, p^{3 g-h} d^{\prime}\right)$ defined by $\psi(\bar{f})=\overline{f_{p}}$ is a one-to-one correspondence. (We may also denote $\psi$ by $\psi_{p}$ or $\psi^{h}$.)

If $A_{f}$ and $A_{f_{p}}$ are the matrices of $f$ and $f_{p}$, respectively, then

$$
A_{f_{p}}=P A_{f} P, \quad \text { where } P=\left[\begin{array}{ccc}
p^{-g / 2} & 0 & 0 \\
0 & p^{(3 g-2 h) / 2} & 0 \\
0 & 0 & p^{g / 2}
\end{array}\right] \text {. }
$$

So

$$
d_{f_{p}}=d_{f} \operatorname{det}(P)^{2}=p^{h} d^{\prime} p^{3 g-2 h}=p^{3 g-h} d^{\prime} .
$$

To show that $N_{f_{p}}=N_{f}$, note that $N_{f} A_{f_{p}}^{-1}=P^{-1} A_{\phi} P^{-1}$, where $\phi$ is the reciprocal of $f$. With $f$ as given, it is not hard to see that

$$
\phi=\left(\begin{array}{ccc}
\alpha & p^{2 g-h} \beta & p^{g} \gamma \\
p^{g} \rho & p^{g} \sigma & p^{2 g-h} \tau
\end{array}\right),
$$

with $\alpha, \beta, \gamma, \rho, \sigma$, and $\tau$ integers, $p \nmid \alpha \beta \gamma$. Then $P^{-1} A_{\phi} P^{-1}$ is the matrix of

$$
\left(\begin{array}{ccc}
p^{g} \alpha & p^{h-g} \beta & \gamma \\
p^{h-g} \rho & p^{g} \sigma & p^{g} \tau
\end{array}\right)
$$

which must be primitive. So $N_{f} A_{f_{p}}^{-1}$ is primitive, and $N_{f_{p}}=N_{f}$ by definition. Thus $\overline{f_{p}}$ is an element of $C\left(N, p^{3 g-h} d^{\prime}\right)$.

Next we show that $\psi$ is a well-defined function. Suppose that $f$ and $F$ are representatives of the same class in $C\left(N, p^{h} d^{\prime}\right)$, and that

$$
f=\left(\begin{array}{ccc}
p^{g} a & p^{h-g} b & c \\
p^{h-g_{r}} & p^{g} S & p^{g} t
\end{array}\right) \text { and } F=\left(\begin{array}{ccc}
p^{g} A & p^{h-g} B & C \\
p^{h-g} R & p^{g} S & p^{g} T
\end{array}\right) \text {, }
$$

with $p \nmid a c A C$, and $p \nmid b B$ if $g<h<2 g$. So $A_{F}=U A_{f} U^{t}$ for some unimodular matrix $U$. We want to show that $f_{p} \sim F_{p}$. We know that $A_{F_{p}}=$ 
$P A_{F} P$ and $A_{f_{p}}=P A_{f} P$, so $A_{F_{p}}=\left(P U P^{-1}\right) A_{f_{p}}\left(P U P^{-1}\right)^{t}$ (since $P=P^{t}$ ). Clearly, $\operatorname{det}\left(P U P^{-1}\right)=\operatorname{det}(U)= \pm 1$. If we can show that the entries of $P U P^{-1}$ are integers, then it is unimodular, and $f_{p}$ and $F_{p}$ are equivalent.

If $U=\left[u_{i j}\right]$, then one can see that

$$
P U P^{-1}=\left[\begin{array}{ccc}
u_{11} & u_{12} p^{h-2 g} & u_{13} p^{-g} \\
u_{21} p^{2 g-h} & u_{22} & u_{23} p^{g-h} \\
u_{31} p^{g} & u_{32} p^{h-g} & u_{33}
\end{array}\right],
$$

so we would like to show that $p^{2 g-h}\left|u_{12}, p^{g}\right| u_{13}$, and $p^{h-g} \mid u_{23}$. This can be seen by looking closely at the implications of the equation $A_{F}=U A_{f} U^{t}$, as given in (3) above. For example, we have that $C=f\left(u_{31}, u_{32}, u_{33}\right)$, so that $C \equiv c u_{33}^{2}\left(\bmod p^{h-g}\right)$. If $h>g$, it follows that $p \nmid u_{33}$. Then one can show that

$$
p^{h-g} R \equiv 2 c u_{33} u_{23} \quad \text { and } \quad p^{g} S \equiv 2 c u_{33} u_{13} \quad\left(\bmod p^{h-g}\right) .
$$

So $p^{h-g} \mid u_{23}$ and $p^{h-g} \mid u_{13}$. If $h=2 g$, then this is all that we need to show.

Suppose that $2 g>h \geq \frac{3}{2} g$. We then have that

$$
p^{h-g} B \equiv p^{h-g} b u_{22}^{2}+c u_{23}^{2}+p^{h-g} r u_{22} u_{23} \equiv p^{h-g} b u_{22}^{2} \quad\left(\bmod p^{g}\right),
$$

since $2(h-g) \geq g$. Since in this case $p \nmid b B$, it follows that $p \nmid u_{22}$. Now

$$
p^{g} T \equiv 2 p^{h-g} b u_{22} u_{12} \quad\left(\bmod p^{g}\right),
$$

so we see that $p^{2 g-h} \mid u_{12}$. Finally,

$$
p^{g} S \equiv 2 c u_{33} u_{13} \quad\left(\bmod p^{g}\right),
$$

so $p^{g} \mid u_{13}$. So $P U P^{-1}$ is unimodular if $2 g \geq h \geq \frac{3}{2} g$.

Now if $h<\frac{3}{2} g$, consider the reciprocals of $f$ and $F$, say $\phi$ and $\Phi$. If $A_{F}=U A_{f} U^{t}$, then $A_{\Phi}=V A_{\phi} V^{t}$ with $V=\left(U^{-1}\right)^{t}$. We can show, by methods similar to those above, that $P^{-1} V P$ is unimodular. But then

$$
\left(P^{-1} V P\right)^{-1}=P^{-1} V^{-1} P=P^{-1} U^{t} P=\left(P U P^{-1}\right)^{t}
$$

is unimodular, so $P U P^{-1}$ is unimodular.

So $\psi^{h}$ is a well-defined function from $C\left(N, p^{h} d^{\prime}\right)$ to $C\left(N, p^{3 g-h} d^{\prime}\right)$. But then it is clear that $\psi^{3 g-h}$ provides an inverse for $\psi^{h}$. So each such function is a one-to-one correspondence and the proof of Theorem 4 is complete.

There is a similar result for $p=2$.

Theorem 5. Let $N$ and $d$ be given by equations (4) and (5). Suppose that $2^{g} \| N$ and $2^{h} \| d$. Write $d$ as $2^{h} d^{\prime}$. Then there is a one-to-one correspondence $\psi$ between $C\left(N, 2^{h} d^{\prime}\right)$ and $C\left(N, 2^{3 g-h-2} d^{\prime}\right)$. This correspondence is defined by $\psi(\bar{f})=\bar{f}_{2}$, where we may assume that $f$ is as given below and then define $f_{2}$ accordingly:

If $h=g-2$, then

$$
f=\left(\begin{array}{ccc}
2^{g-2} a & b & c \\
r & 2^{g-1} s & 2^{g-1} t
\end{array}\right), \quad f_{2}=\left(\begin{array}{ccc}
a & 2^{g} b & 2^{g} c \\
2^{g} r & 2^{g} s & 2^{g} t
\end{array}\right) .
$$

If $g \leq h \leq 2 g-2$, then

$f=\left(\begin{array}{ccc}2^{g-2} a & 2^{h-g} b & c \\ 2^{h-g+1} r & 2^{g-1} s & 2^{g-1} t\end{array}\right), \quad f_{2}=\left(\begin{array}{ccc}a & 2^{2 g-h-2} b & 2^{g-2} c \\ 2^{g-1} r & 2^{g-1} s & 2^{2 g-h-1} t\end{array}\right)$. 
If $h=2 g$, then

$$
f=\left(\begin{array}{ccc}
2^{g} a & 2^{g} b & c \\
2^{g} r & 2^{g} s & 2^{g} t
\end{array}\right), \quad f_{2}=\left(\begin{array}{ccc}
a & b & 2^{g-2} c \\
2^{g-1} r & 2^{g-1} s & t
\end{array}\right) .
$$

The proof of Theorem 5 is similar to that of Theorem 4 (and Lemma 2), with the extra care which the prime 2 usually requires. For the computational purposes which are our focus, however, we can see that the effect of these correspondences may be more easily obtained by the use of the reciprocal correspondence of Theorem 3. So we will omit the proof of this theorem.

We combine and summarize the results of this section as:

Corollary 2. Let $N$ and $d$ be given by equations (4) and (5), and let $e=$ $2^{e_{0}} p_{1}^{e_{1}} \cdots p_{k}^{e_{k}}$. Then there is a one-to-one correspondence between $C(N, d)$ and $C(N, e)$ if for all $1 \leq i \leq k, e_{i}=d_{i}$ or $e_{i}=3 n_{i}-d_{i}$, and if $e_{0}=d_{0}$ or $e_{0}=3 n_{0}-d_{0}-2$. Thus, representatives of all classes of ternary forms of level $N$ can be obtained by applying a sequence of the functions $\psi_{p}$ to the classes in $C(N, d)$ with $d_{0} \leq \frac{3}{2} n_{0}-1$, and $d_{i} \leq \frac{3}{2} n_{i}$ for $1 \leq i \leq k$.

For example, consider $N=60=2^{2} \cdot 3 \cdot 5$. By Theorem 2 , there are twelve potential discriminants for ternary forms of level 60 . But we need only find the reduced forms for two of them: $d=15$ and $d=60$. Applying the map $\psi_{3}$ to the set $C(60,15)$ gives us the entire set $C(60,45)$. Applying $\psi_{5}$ to these two sets yields all of $C(60,75)$ and $C(60,225)$. Taking the reciprocals of all of these forms gives us all of the elements in $C(60, d)$ for $d=3600,1200$, 720, and 240. (We could obtain the same sets by applying the map $\psi_{2}$ at this point.) Applying $\psi_{3}$ to $C(60,60)$ gives us all of $C(60,180)$. We may take reciprocals of those forms to obtain all forms in $C(60,900)$ and $C(60,300)$. Thus, we have representatives (but not in general the reduced forms) of all classes of ternary forms with level 60 . Note that if $N / 4$ is squarefree, then only two discriminant values, $d=N / 4$ and $d=N$, need to be considered.

The process outlined here can be effectively computed for many values of $N$. Using this method, along with an algorithm for finding the reduced form in the class of a given positive definite ternary form, the author has found all reduced forms with level $N \leq 1500$, and all with $N \leq 4000$ for which $N / 4$ is squarefree $(349,186$ forms in all). In Table 1 in the supplement to this issue, we present a small part of these results-the reduced forms with level $N \leq 100$. Note that in that table, the forms are ordered so as to preserve the effect of the $\psi$-maps defined above. That is, suppose that $p^{g} \| N$ and that $d=p^{h} d^{\prime}$ with $\operatorname{gcd}\left(p, d^{\prime}\right)=1$. If the forms listed to the right of $d$ in Table 1 are in order

$f_{1}, \ldots, f_{n}$, then the forms listed to the right of $d_{1}=p^{3 g-h} d^{\prime}\left(p^{3 g-h-2} d^{\prime}\right.$, if $p=2)$ are in order $\psi_{p}\left(f_{1}\right), \ldots, \psi_{p}\left(f_{n}\right)$.

\section{LEVELS AND GENERA}

In this section, we return to the application mentioned in the introduction, that is, the relation between (ternary) quadratic forms and (weight $3 / 2$ ) modular and cusp forms. We first consider another classification of quadratic forms. Two integral quadratic forms are said to be semi-equivalent if they are equivalent over the $p$-adic integers for all primes $p$, and are equivalent over the real numbers (see [3] or [6] for more details). Semi-equivalent forms are said to be 
in the same genus (pl. genera) of forms: Equivalent forms are semi-equivalent, so we may speak of a class of forms as belonging to a genus.

For ternary forms, semi-equivalence can be tested as follows. Let $f$ be a ternary form and $\phi$ its reciprocal, as given in equations (1) and (2) above. Let $m$ and $\mu$ be the divisors of these forms. We can assume, by Proposition 2, that $a$ and $\gamma$ are relatively prime to $m \mu$. If $p$ is an odd prime and $p \mid m$, define a symbol $(f / p)$ to be the Legendre symbol $(a / p)$. Similarly, if $p \mid \mu$, define $(\phi / p)$ to be $(\gamma / p)$. If $16 \mid m$, let $(f / 4)=(-1)^{(a-1) / 2}$. If $32 \mid m$, let $(f / 8)=(-1)^{\left(a^{2}-1\right) / 8}$. Define $(\phi / 4)$ and $(\phi / 8)$ analogously if $\mu$ is divisible by 16 or 32 . We will refer to these symbols (whichever ones are defined) as the collection of genus symbols for $f$.

Proposition 4 [3, pp. 378-384; 4, pp. 51, 52]. Let $f$ and $g$ be primitive, positive definite ternary forms. Then $f$ and $g$ are in the same genus if and only if they have the same discriminant and level (and thus the same values of $m$ and $\mu$ ) and the same collection of genus symbols.

Remarks. The definition of genus symbols given here is adapted from the definition of characters in [4]. That genus symbols are well defined can be shown directly; the proof is omitted. Proposition 4 can be established by showing that two ternary forms have the same genus symbols if and only if they have the same $p$-adic symbols as defined in [3]. It can also be shown directly that semi-equivalent forms have the same discriminant and level. Note that by Proposition 4, it is obvious that the reciprocals of semi-equivalent forms are semi-equivalent. Similarly, one can show that the maps of Theorems 4 and 5 are genus-preserving, that is, if two classes of forms are in the same genus, then so are $\psi_{p}$ applied to those classes, as defined.

We note also a result on the existence of forms having a particular collection of genus symbols.

Proposition 5 [6, Theorem 46]. Let $m=2^{m_{0}} p_{1}^{m_{1}} \cdots p_{k}^{m_{k}}$ and $\mu=2^{\mu_{0}} p_{1}^{\mu_{1}} \cdots p_{k}^{\mu_{k}}$ be two integers subject to the conditions that $m_{0} \neq 1, \mu_{0} \neq 1$, and $m_{0}+\mu_{0} \geq 4$. (Each $p_{i}$ is a distinct odd prime; we do not assume that $m_{i}$ and $\mu_{i}$ are both positive.) Let $h$ (respectively $\eta$ ) equal \pm 1 as $m / 2^{m_{0}}$ (respectively $\mu / 2^{\mu_{0}}$ ) is congruent to $\pm 1(\bmod 4)$. For $i=1, \ldots, k$, let the symbols $\left(f / p_{i}\right)$ and $\left(\phi / p_{i}\right)$ be chosen independently as \pm 1 ; similarly choose $(f / 4),(f / 8),(\phi / 4)$, and $(\phi / 8)$.

Then there is a primitive, positive definite ternary form $f$ with reciprocal $\phi$ so that $f$ has discriminant $d=m^{2} \mu / 16$, level $N=m \mu / 4$, and genus symbols

$$
\begin{array}{lll}
\left(\frac{f}{p_{i}}\right) \quad \text { if } m_{i}>0, & \left(\frac{f}{4}\right) \quad \text { if } m_{0} \geq 4, & \left(\frac{f}{8}\right) \quad \text { if } m_{0} \geq 5, \\
\left(\frac{\phi}{p_{i}}\right) \quad \text { if } \mu_{i}>0, & \left(\frac{\phi}{4}\right) \quad \text { if } \mu_{0} \geq 4, & \left(\frac{\phi}{8}\right) \quad \text { if } \mu_{0} \geq 5,
\end{array}
$$

if and only if the following conditions hold:

$$
\begin{aligned}
\left(\frac{f}{8}\right)^{m_{0}} & \left(\frac{\phi}{8}\right)^{\mu_{0}} \prod_{i=1}^{k}\left(\frac{f}{p_{i}}\right)^{m_{i}}\left(\frac{\phi}{p_{i}}\right)^{\mu_{i}} \\
= & (-1)^{((f / 4)+\eta)((\phi / 4)+h) / 4}(-1)^{(h+1)(\eta+1) / 4},
\end{aligned}
$$


and

$$
\left(\frac{\phi}{4}\right)=-h \quad \text { if } m_{0}=0, \quad\left(\frac{f}{4}\right)=-\eta \quad \text { if } \mu_{0}=0 .
$$

Remarks. The notation is again adapted from that of Dickson [4, pp. 51-54]. It can be shown directly, by methods similar to those of Lemma 1, that conditions (6) and (7) hold for any ternary form $f$. (Lemma 1 is in fact a special case of Proposition 5.) The existence of ternary forms subject to these conditions follows from Theorem 46 in [6].

If $f$ is a positive definite ternary quadratic form of level $N$ and discriminant $d$, then $\theta(f)$, as defined in the introduction, is in $M_{3 / 2}\left(N, \chi_{d}\right)$. Recall that $\chi_{d}$, a Dirichlet character modulo $N$, depends only on the squarefree part of $d$. In keeping up the connection between modular forms and quadratic forms, we will say that a ternary form $f$ has character $q$ if $d=q r^{2}$ and $q$ is squarefree. If $f$ has level $N$, its character is a squarefree divisor of $N / 4$.

If $f_{1}$ and $f_{2}$ are equivalent forms, then $\theta\left(f_{1}\right)=\theta\left(f_{2}\right)$. Let $c=c_{q}(N)$ be the number of classes of ternary forms having level $N$ and character $q$. We thus have $c$ forms in $M_{3 / 2}\left(N, \chi_{q}\right)$. These forms might not be linearly independent, but $c$ provides an upper limit on the number of independent modular forms which arise directly from quadratic forms.

If $f_{1}$ and $f_{2}$ are semi-equivalent ternary forms of level $N$ and character $q$, then $\theta\left(f_{1}\right)-\theta\left(f_{2}\right)$ is in $S_{3 / 2}\left(N, \chi_{q}\right)$. Let $g=g_{q}(N)$ be the number of genera of ternary forms with level $N$ and character $q$. Of course, $g \leq c$ in all cases. Suppose that a genus of forms contains $n$ classes, say with $f_{1}, f_{2}, \ldots, f_{n}$ as class representatives. Then $\theta\left(f_{1}\right)-\theta\left(f_{2}\right), \ldots, \theta\left(f_{1}\right)-\theta\left(f_{n}\right)$ are cusp forms which might be independent. Any other difference, though, is easily seen to be a linear combination of these $n-1$ forms. Thus there is a maximum of $n-1$ linearly independent cusp forms arising directly from this genus.

Now suppose that the $c$ classes of level $N$ and character $q$ are partitioned into the corresponding $g$ genera so that the first genus contains $c_{1}$ classes, the second genus contains $c_{2}$ classes, and so on. Then the maximum number of linearly independent cusp forms which can be constructed from these classes is

$$
\left(c_{1}-1\right)+\left(c_{2}-1\right)+\cdots+\left(c_{g}-1\right)=\sum_{i=1}^{g} c_{i}-\sum_{i=1}^{g} 1=c-g .
$$

Let $s_{q}(N)=c_{q}(N)-g_{q}(N)$.

We can calculate $g_{q}(N)$ for all values of $N$ and $q$. First let $g(N, d)$ denote the number of genera of forms of level $N$ and discriminant $d$.

Lemma 3. Let $N$ and $d$ be given by equations (4) and (5). For $1 \leq i \leq k$, let

$$
r_{i}= \begin{cases}1 & \text { if } d_{i}=n_{i} \text { or } d_{i}=2 n_{i}, \\ 2 & \text { if } n_{i}<d_{i}<2 n_{i} .\end{cases}
$$

Let $r=r_{1}+\cdots+r_{k}$. Then $g(N, d)=c \cdot 2^{r}$, where $c$ is defined as follows:

(I) If $N$ and $d$ are both squares, then

$$
c= \begin{cases}1 & \text { if } 2 n_{0}-4 \leq d_{0} \leq n_{0}+2 \\ 4 & \text { if } n_{0}+4 \leq d_{0} \leq 2 n_{0}-6 \\ 2 & \text { otherwise }\end{cases}
$$


(II) If $N$ and $d$ are not both squares, then

$$
c= \begin{cases}1 / 2 & \text { if } n_{0}=2, d_{0}=0 \text { or } 4, \\ 1 & \text { if } n_{0}=2, d_{0}=2 ; n_{0}=3, d_{0}=3 \text { or } 4 ; n_{0}=4, d_{0}=5, \\ 3 / 2 & \text { if } n_{0}=4, d_{0}=4 \text { or } 6, \\ 2 & \text { if } n_{0}=5, d_{0}=6 \text { or } 7 ; n_{0}=6, d_{0}=8 .\end{cases}
$$

If $n_{0}$ and $d_{0}$ are not among these exceptional cases, then

$$
c=\left\{\begin{array}{l}
1 \text { if } d_{0}=n_{0}-2 \text { or } 2 n_{0} \\
3 \text { if } d_{0}=n_{0} \text { or } 2 n_{0}-2, \\
4 \text { if } d_{0}=n_{0}+1, n_{0}+2,2 n_{0}-4, \text { or } 2 n_{0}-3 \\
8 \text { if } n_{0}+3 \leq d_{0} \leq 2 n_{0}-5
\end{array}\right.
$$

Proof. We want to count the number of different collections of genus symbols which are allowed by conditions (6) and (7) of Proposition 5. Notice that in that statement, some symbols are defined, and may play a part in equation (6), which are not part of the collection of genus symbols. We will say that a symbol is "relevant" if it is in fact a genus symbol. For example, $\left(f / p_{i}\right)$ is relevant if $n_{i}<d_{i}$, and $\left(\phi / p_{i}\right)$ is relevant if $d_{i}<2 n_{i}$. So $r$ is the number of relevant symbols involving the odd primes. Call these the "odd" symbols. For the others, $(f / 4)($ resp. $(f / 8))$ is relevant if $d_{0} \geq n_{0}+2$ (resp. $\left.n_{0}+3\right) ;(\phi / 4)$ (resp. $(\phi / 8))$ is relevant if $d_{0} \leq 2 n_{0}-4$ (resp. $2 n_{0}-5$ ).

Case I. If $N$ and $d$ are squares, then so are $m$ and $\mu$, so we have that $h=1=\eta$. Each $m_{i}$ and $\mu_{i}$ is even, so equation (6) becomes

$$
1=-(-1)^{((f / 4)+1)((\phi / 4)+1) / 4} \text {. }
$$

By Lemma 1, neither $m$ nor $\mu$ can be odd, so condition (7) does not apply in this case. Equation (6) reduces to requiring only that $(f / 4)=1=(\phi / 4)$. Otherwise, we see that the $r$ odd symbols can be chosen independently, as can $(f / 8)$ and $(\phi / 8)$. So the number of possibilities for these choices is:

$2^{r}$ if neither $(f / 8)$ nor $(\phi / 8)$ is relevant,

$2 \cdot 2^{r}$ if only one of $(f / 8)$ and $(\phi / 8)$ is relevant,

$4 \cdot 2^{r}$ if both $(f / 8)$ and $(\phi / 8)$ are relevant.

With the facts noted in the previous paragraph, and the fact that here $n_{0}$ and $d_{0}$ are both even, we get the result of the theorem.

Case II. Suppose that $N$ and $d$ are not both squares. We can rewrite equation (6) as

$$
\begin{aligned}
\left(\frac{f}{8}\right)^{d_{0}-n_{0}}\left(\frac{\phi}{8}\right)^{d_{0}} \prod_{i=1}^{k}\left(\frac{f}{p_{i}}\right)^{d_{i}-n_{i}}\left(\frac{\phi}{p_{i}}\right)^{d_{i}} & \\
= & (-1)^{((f / 4)+\eta)((\phi / 4)+h) / 4}(-1)^{(h+1)(\eta+1) / 4} .
\end{aligned}
$$

Note that if $n_{0}$ and $d_{0}$ are both even, then in this case we must have that $n_{i}$ or $d_{i}$ is odd for some $1 \leq i \leq k$. So then $r>0$, and it makes sense to speak of choosing $r-1$ odd symbols in a particular way. (In all other cases, we do not assume that $k>0$.) 
(1) Let $d_{0}=n_{0}-2$. Here, $(\phi / 4)$ is relevant, as is $(\phi / 8)$ if $n_{0} \geq 3$. Neither $(f / 4)$ nor $(f / 8)$ is relevant. In this case, $m$ is odd, so we know that $(\phi / 4)=-h$. Equation $(6)$ becomes

$$
\left(\frac{\phi}{8}\right)^{n_{0}} \prod_{i=1}^{k}\left(\frac{f}{p_{i}}\right)^{d_{i}-n_{i}}\left(\frac{\phi}{p_{i}}\right)^{d_{i}}=(-1)^{(h+1)(\eta+1) / 4}
$$

If $n_{0}$ is odd, then we may choose the $r$ odd symbols as we like; the value of $(\phi / 8)$ is then determined by this equation. If $n_{0}$ is even, then the value of $(\phi / 8)$ plays no part in equation (6). We can choose $(\phi / 8)$ and $r-1$ of the odd symbols independently. The last odd symbol is then determined. So if $n_{0} \geq 3$, we have $r$ independent choices for the relevant symbols, for a total of $2^{r}$ possibilities. If $n_{0}=2$, then there are $2^{r-1}=\frac{1}{2} \cdot 2^{r}$ possibilities for the collection of genus symbols.

The case in which $d_{0}=2 n_{0}$ is the same (with $f$ and $\phi$ interchanged).

(2) Let $d_{0}=n_{0}$. Here, $(\phi / 4)$ is relevant if $n_{0} \geq 4,(\phi / 8)$ if $n_{0} \geq 5$. Neither $(f / 4)$ nor $(f / 8)$ is relevant. Equation (6) becomes

$$
\left(\frac{\phi}{8}\right)^{n_{0}} \prod_{i=1}^{k}\left(\frac{f}{p_{i}}\right)^{d_{i}-n_{i}}\left(\frac{\phi}{p_{i}}\right)^{d_{i}}=(-1)^{((f / 4)+\eta)((\phi / 4)+h) / 4}(-1)^{(h+1)(\eta+1) / 4}
$$

Suppose first that $n_{0} \geq 4$. If $(\phi / 4)=-h$, then the right-hand side of equation $(6 a)$ is $(-1)^{(h+1)(\eta+1) / 4}$, which determines the left-hand side. As in subcase (1) above, we have $r$ independent choices for $(\phi / 8)$ and the odd symbols. (Again, which ones we can choose depends on the parity of $\left.n_{0}.\right)$ On the other hand, if $(\phi / 4)=h$, then the right-hand side is $\pm(-1)^{(h+1)(\eta+1) / 4}$ depending on the value of $(f / 4)$. We have $r+1$ free choices for the odd symbols and for $(\phi / 8)$. $\left((f / 4)\right.$ is then determined but is not relevant.) So if $n_{0} \geq 5$, then there is a total of $2^{r}+2^{r+1}=3 \cdot 2^{r}$ possibilities for the relevant symbols. If $n_{0}=4$, then $(\phi / 8)$ is not relevant, so the total number of possibilities is $2^{r-1}+2^{r}=\frac{3}{2} \cdot 2^{r}$.

Now if $n_{0}<4$, then neither $(f / 4)$ nor $(\phi / 4)$ is relevant. By choosing their values as we like, we have $r$ free choices for the odd symbols. So there are $2^{r}$ possibilities if $n_{0}=3$ or $n_{0}=2$.

The case in which $d_{0}=2 n_{0}-2$ is the same.

(3) Let $d_{0}=n_{0}+1$. Here, $(\phi / 4)$ is relevant if $n_{0} \geq 5,(\phi / 8)$ if $n_{0} \geq 6$. Neither $(f / 4)$ nor $(f / 8)$ is relevant. Equation $(6)$ becomes

$$
\left(\frac{f}{8}\right)\left(\frac{\phi}{8}\right)^{n_{0}+1} \prod_{i=1}^{k}\left(\frac{f}{p_{i}}\right)^{d_{i}-n_{i}}\left(\frac{\phi}{p_{i}}\right)^{d_{i}}=(-1)^{((f / 4)+\eta)((\phi / 4)+h) / 4}(-1)^{(h+1)(\eta+1) / 4}
$$

By choosing $(f / 8)$ as we like, we have free choices for each of the relevant symbols. So the total number of possibilities is $2^{r+2}=4 \cdot 2^{r}$ if $n_{0} \geq 6$, $2^{r+1}=2 \cdot 2^{r}$ if $n_{0}=5$, and $2^{r}$ if $n_{0} \leq 4$.

The case in which $d_{0}=2 n_{0}-3$ is the same.

(4) Let $d_{0}=n_{0}+2$. Now $(f / 4)$ is relevant, but $(f / 8)$ is not; $(\phi / 4)$ is relevant if $n_{0} \geq 6,(\phi / 8)$ if $n_{0} \geq 7$. Equation (6) becomes (6a) again. If 
$n_{0} \geq 6$, then choose $(f / 4)$ and $(\phi / 4)$ independently. The left-hand side of equation (6a) is then determined. There are $r$ free choices for $(\phi / 8)$ and the odd symbols. So if $n_{0} \geq 7$, then there is a total of $2^{r+2}=4 \cdot 2^{r}$ possibilities for the relevant symbols. If $n_{0}=6$, there are $2 \cdot 2^{r}$ total possibilities. The cases in which $n_{0} \leq 5$ are already accounted for.

The case in which $d_{0}=2 n_{0}-4$ is the same.

(5) Finally, let $n_{0}+3 \leq d_{0} \leq 2 n_{0}-5$. Here, $(f / 4),(f / 8),(\phi / 4)$, and $(\phi / 8)$ are all relevant. Choosing $(f / 4)$ and $(\phi / 4)$ determines the left-hand side of equation (6). We can choose $r+1$ of the remaining symbols freely. The total number of possible collections is $2^{r+3}=8 \cdot 2^{r}$.

So the proof of Lemma 3 is complete.

Notice that the number of genera of ternary forms of level $N$ and discriminant $d$ is positive in all cases listed. So the number of such classes must be positive as well. This proves the remark which concludes $\S 1$.

With $N$ given by equation (4), let $q=2^{q_{0}} p_{1}^{q_{1}} \cdots p_{k}^{q_{k}}$ with each $q_{i}$ equal to 0 or 1 , and $q_{0}=0$ if $n_{0}=2$. So $q$ is a possible character for a ternary form of level $N$. We can now calculate $g_{q}(N)$ as

$$
g_{q}(N)=\sum_{d} g(N, d)=\sum_{d} c \cdot 2^{r}
$$

where $c$ and $r$ are as given in Lemma 3, and the sum is taken over all $d$, given by equation (5), for which $\operatorname{sf}(d)=q$. A sum over all such $d$ can be viewed as a sum over $(k+1)$-tuples $\left(d_{0}, d_{1}, \ldots, d_{k}\right)$ for which $d_{i} \equiv q_{i}(\bmod 2)$.

Let $r_{i}$ be given as in the statement of Lemma 3. Then we have

$$
g_{q}(N)=\sum_{d} c \cdot 2^{r}=\sum_{\left(d_{0}, \ldots, d_{k}\right)} c \cdot 2^{r_{1}} \cdots 2^{r_{k}} .
$$

But $c$ depends only on $d_{0}$ (once it is determined whether Case I or Case II applies), while $2^{r_{i}}$ depends only on $d_{i}$. So we can see that

$$
g_{q}(N)=\sum_{d_{0}} c \cdot \sum_{d_{1}} 2^{r_{1}} \cdots \sum_{d_{k}} 2^{r_{k}}
$$

Theorem 6. Let $N$ be given by equation (4) and let $q$ be a squarefree divisor of $N / 4$. Let $g_{q}(N)$ be the number of genera of positive definite ternary forms having level $N$ and character $q$. Then

$$
g_{q}(N)=C \prod_{i=1}^{k}\left(2 n_{i}\right)
$$

where $C$ is a constant defined as follows:

(I) If $N$ is a square and $q=1$, then

$$
C= \begin{cases}1 & \text { if } n_{0}=2, \\ 2 & \text { if } n_{0}=4 \\ 5 & \text { if } n_{0}=6 \\ 2\left(n_{0}-4\right) & \text { if } n_{0} \geq 8\end{cases}
$$


(II) If $N$ is not a square or $q \neq 1$, then

$$
C= \begin{cases}2 & \text { if } n_{0}=2 \text { or } 3, \\ 1 & \text { if } n_{0}=4 \text { and } q \text { is even, } \\ 5 & \text { if } n_{0}=4 \text { and } q \text { is odd, } \\ 6 & \text { if } n_{0}=5, \\ 10 & \text { if } n_{0}=6 \text { and } q \text { is odd, } \\ 4\left(n_{0}-4\right) & \text { if } n_{0}=6 \text { and } q \text { is even, or if } n_{0} \geq 7\end{cases}
$$

Proof. In light of the remarks above, we need only show that

$$
\sum_{d_{i}} 2^{r_{i}}=2 n_{i} \text { for } i=1, \ldots, k, \text { and } \sum_{d_{0}} c=C .
$$

For $i=1, \ldots, k$, we will consider three cases:

(i) If $n_{i}$ is even and $q_{i}=0$, then the possibilities for $d_{i}$ are $n_{i}, n_{i}+$ $2, \ldots, 2 n_{i}-2$, and $2 n_{i}$. Then $r_{i}=1$ for $d_{i}=n_{i}$ and $d_{i}=2 n_{i}$, and $r_{i}=2$ in all other cases. Notice that $n_{i}+2 \leq d_{i} \leq 2 n_{i}-2$ for $\left(n_{i}-2\right) / 2$ even values of $d_{i}$. Thus

$$
\sum_{d_{i}} 2^{r_{i}}=2^{1}+\frac{n_{i}-2}{2}\left(2^{2}\right)+2^{1}=4+2\left(n_{i}-2\right)=2 n_{i} .
$$

(ii) If $n_{i}$ is even and $q_{i}=1$, then $d_{i}=n_{i}+1, n_{i}+3, \ldots, 2 n_{i}-1$. There are $n_{i} / 2$ such values of $d_{i}$, and $r_{i}=2$ in each case, so

$$
\sum_{d_{i}} 2^{r_{i}}=\frac{n_{i}}{2}\left(2^{2}\right)=2 n_{i}
$$

(iii) Suppose that $n_{i}$ is odd. If $q_{i}=0$, then $d_{i}=n_{i}+1, \ldots, 2 n_{i}-2,2 n_{i}$. If $q_{i}=1$, then $d_{i}=n_{i}, n_{i}+2, \ldots, 2 n_{i}-1$. In either case, there is one value of $d_{i}$ for which $r_{i}=1$ and $\left(n_{i}-1\right) / 2$ values for which $r_{i}=2$. So

$$
\sum_{d_{i}} 2^{r_{i}}=2^{1}+\frac{n_{i}-1}{2}\left(2^{2}\right)=2+2\left(n_{i}-1\right)=2 n_{i} .
$$

Now let $c$ be defined as in Lemma 3.

Case I. Suppose that $N$ is a square and that $q=1$ (so that $d$ is also a square). In particular, $n_{0}$ and $d_{0}$ are both even. If $n_{0} \geq 8$, then there are the following possibilities for $d_{0}: n_{0}, n_{0}+2,2 n_{0}-4,2 n_{0}-2$, and for $\left(n_{0}-8\right) / 2$ values, $n_{0}+4 \leq d_{0} \leq 2 n_{0}-6$. By Lemma 3 , for the first four values, $c=2$, and for the others, $c=4$. So we have that

$$
C=2+2+2+2+\frac{n_{0}-8}{2}(4)=2\left(n_{0}-4\right) \text {. }
$$

For the other values of $n_{0}$, we have

$$
\begin{array}{ll}
n_{0}=6 \Rightarrow d_{0}=6,8, \text { or } 10 & \Rightarrow C=2+1+2=5, \\
n_{0}=4 \Rightarrow d_{0}=4 \text { or } 6 & \Rightarrow C=1+1=2, \\
n_{0}=2 \Rightarrow d_{0}=2 & \Rightarrow C=1 .
\end{array}
$$

Case II. Suppose that $N$ is not a square or that $q \neq 1$ (that is, $N$ and $d$ are not both squares). We consider three subcases here. 
(i) Suppose that $n_{0}$ and $d_{0}$ are even, so that $q$ is odd. If $n_{0} \geq 8$, then $d_{0}=n_{0}-2, n_{0}, n_{0}+2,2 n_{0}-4,2 n_{0}-2,2 n_{0}$, and for $\left(n_{0}-8\right) / 2$ values, $n_{0}+4 \leq d_{0} \leq 2 n_{0}-6$. Then by Lemma 3 ,

$$
C=1+3+4+4+3+1+\frac{n_{0}-8}{2}(8)=4\left(n_{0}-4\right) .
$$

Otherwise, we have

$$
\begin{array}{ll}
n_{0}=6 \Rightarrow d_{0}=4,6,8,10, \text { or } 12 & \Rightarrow C=1+3+2+3+1=10, \\
n_{0}=4 \Rightarrow d_{0}=2,4,6, \text { or } 8 & \Rightarrow C=1+\frac{3}{2}+\frac{3}{2}+1=5, \\
n_{0}=2 \Rightarrow d_{0}=0,2, \text { or } 4 & \Rightarrow C=\frac{1}{2}+1+\frac{1}{2}=2 .
\end{array}
$$

(ii) Suppose that $n_{0}$ is even, and $d_{0}$ is odd, so that $q$ is even. For $n_{0} \geq 6$, we have that $d_{0}=n_{0}+1,2 n_{0}-3$, and for $\left(n_{0}-6\right) / 2$ values, $n_{0}+3 \leq d_{0} \leq 2 n_{0}-5$. Then

$$
C=4+4+\frac{n_{0}-6}{2}(8)=4\left(n_{0}-4\right) .
$$

If $n_{0}=4$, then $d_{0}=5$ is the only possibility, so $C=1$.

(iii) Suppose that $n_{0}$ is odd. We will assume that $d_{0}$ is even; the case in which $d_{0}$ is odd is similar. If $n_{0} \geq 7$, then $d_{0}=n_{0}+1,2 n_{0}-4,2 n_{0}-2$, $2 n_{0}$, and for $\left(n_{0}-7\right) / 2$ values, $n_{0}+3 \leq d_{0} \leq 2 n_{0}-6$. So then,

$$
C=4+4+3+1+\frac{n_{0}-7}{2}(8)=4\left(n_{0}-4\right) \text {. }
$$

Otherwise,

$$
\begin{aligned}
& n_{0}=5 \Rightarrow d_{0}=6,8, \text { or } 10 \Rightarrow C=2+3+1=6, \\
& n_{0}=3 \Rightarrow d_{0}=4 \text { or } 6 \quad \Rightarrow C=1+1=2 .
\end{aligned}
$$

This completes the proof of Theorem 6 .

General results concerning the values of $c_{q}(N)$ and $s_{q}(N)$ are not apparent. However, combining the results of Theorems 4,5 , and 6 , we can easily establish the following:

Theorem 7. Let $N$ be divisible by 4 , and let $Q=\operatorname{sf}(N / 4)$. Suppose that $r$ is a squarefree divisor of $N / 4$ and that $\operatorname{gcd}(r, Q)=1$. Then

$$
c_{r}(N)=c_{r q}(N) \text { and } s_{r}(N)=s_{r q}(N)
$$

if $q$ is any divisor of $Q$.

In particular, if each prime in the unique factorization of $N / 4$ appears with odd exponent, then the values $c_{q}(N)$ and $s_{q}(N)$ are independent of $q$.

Using the ternary forms listed in Table 1 (see Supplements section), the author has found bases for all spaces of cusp forms of weight $3 / 2$, level $N \leq 100$, and quadratic character. However, for larger values of $N$, the quadratic form method will not suffice in this direct way for construction of such bases. Suppose that $N=4 p$ for some prime $p$. (We restrict our attention to this case because then $S_{3 / 2}(N, \chi)$ does not contain any nontrivial subspaces of the form $S_{3 / 2}(M, \chi)$ with $M<N$.) It can be shown that 


$$
\operatorname{dim} S_{3 / 2}(N, \chi)= \begin{cases}(p-5) / 4 & \text { if } p \equiv 1(\bmod 4), \\ (p-3) / 4 & \text { if } p \equiv 3(\bmod 4)\end{cases}
$$

if $N=4 p$ with $p$ an odd prime, and $\chi=\chi_{1}$ or $\chi=\chi_{p}$ [2, Theorem $2 ; 11$, Theorem A].

In Table 2 in the supplement to this issue, we compare $\operatorname{dim} S_{3 / 2}\left(N, \chi_{q}\right)$ with our calculation of $s_{q}(N)$ for each $N \leq 4000$ with $N / 4$ prime. It is apparent that, for these values, $s_{q}(N)$ does not increase as quickly as does $\operatorname{dim} S_{3 / 2}\left(N, \chi_{q}\right)$. Thus, the question of how useful the quadratic form method is in constructing a basis for a space of modular or cusp forms remains unresolved.

\section{BIBLIOGRAPHY}

1. H. Brandt and O. Intrau, Tabellen reduzierter positiver ternärer quadratischer Formen, Abh. Sächs. Akad. Wiss. Leipzig Math.-Natur. Kl. 45 (1958).

2. H. Cohen and J. Oesterlé, Dimensions des espaces de formes modulaires, Lecture Notes in Math., vol. 627, Springer-Verlag, Berlin, 1977.

3. J. H. Conway and N. J. A. Sloane, Sphere packings, lattices and groups, Springer-Verlag, New York, 1988.

4. L. E. Dickson, Studies in the theory of numbers, The University of Chicago Press, Chicago, 1930.

5. H. Hijikata, A. Pizer, and T. Shemanske, The basis problem for modular forms on $\Gamma_{0}(N)$, Mem. Amer. Math. Soc. No. 82 (1989), 1-159.

6. B. W. Jones, The arithmetic theory of quadratic forms, Math. Assoc. America and Wiley, New York, 1950.

7. N. Koblitz, Introduction to elliptic curves and modular forms, Springer-Verlag, New York, 1984.

8. J. L. Lehman, Rational points on elliptic curves with complex multiplication by the ring of integers in $\mathbb{Q}(\sqrt{-7})$, J. Number Theory 27 (1987), 253-272.

9. B. Schoeneberg, Elliptic modular functions, an introduction, Springer-Verlag, New York, 1974.

10. R. Schulze-Pillot, Thetareihen positiv definiter quadratischer Formen, Invent. Math. 75 (1984), 283-299.

11. J.-P. Serre and H. M. Stark, Modular forms of weight $\frac{1}{2}$, Lecture Notes in Math., vol. 627 , Springer-Verlag, Berlin, 1977.

12. G. Shimura, On modular forms of half-integral weight, Ann. of Math. (2) 97 (1973), 440481 .

Department of Mathematics, Mary Washington College, Fredericksburg, Virginia

E-mail address: llehman@s850.mwc.edu 


\section{Supplement to}

LEVELS OF POSITIVE DEFINITE TERNARY QUADRATIC FORMS

\section{J. LARRY LEHMAN}

\section{TABLE 1}

All Reduced Ternary Forms of Level $N \leq 100$

For each level $N \leq 100$, the reduced forms are grouped by character and by discriminant. A wedge $(V)$ between two forms indicates that those forms are in the same genus.

$\frac{q}{1} \frac{d}{4} \quad\left(\begin{array}{lll}1 & 1 & 1 \\ 0 & 0 & 0\end{array}\right)$

$\begin{array}{ccc}\frac{q}{1} & \frac{d}{16} & \left(\begin{array}{lll}1 & 2 & 2 \\ 0 & 0 & 0\end{array}\right) \\ & 64 & \left(\begin{array}{ccc}3 & 3 & 3 \\ -2 & -2 & -2\end{array}\right) \\ 2 & 2 & \left(\begin{array}{lll}1 & 1 & 1 \\ 1 & 1 & 1\end{array}\right) \\ & 8 & \left(\begin{array}{lll}1 & 1 & 2 \\ 0 & 0 & 0\end{array}\right)\end{array}$

$$
\underline{N}=4
$$

$$
\underline{N=8}
$$


$\stackrel{+}{11}$
$z$

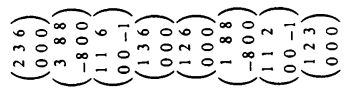

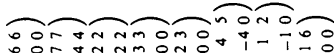

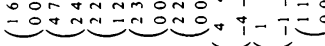

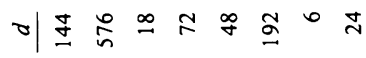

ol $N$ m

그

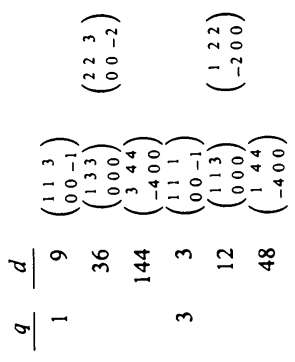

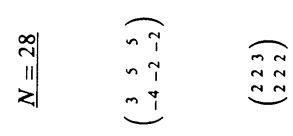

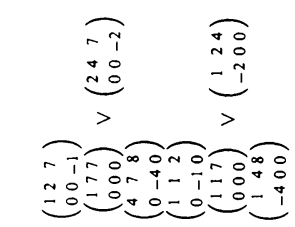

ช|

of

원

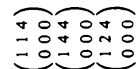

○|ำ

of

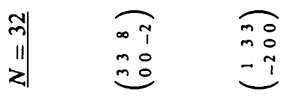

กำ

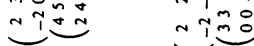

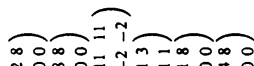

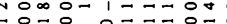

○|

orl

N

이
II

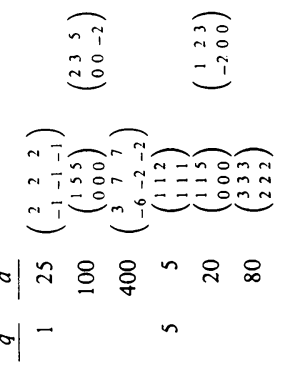



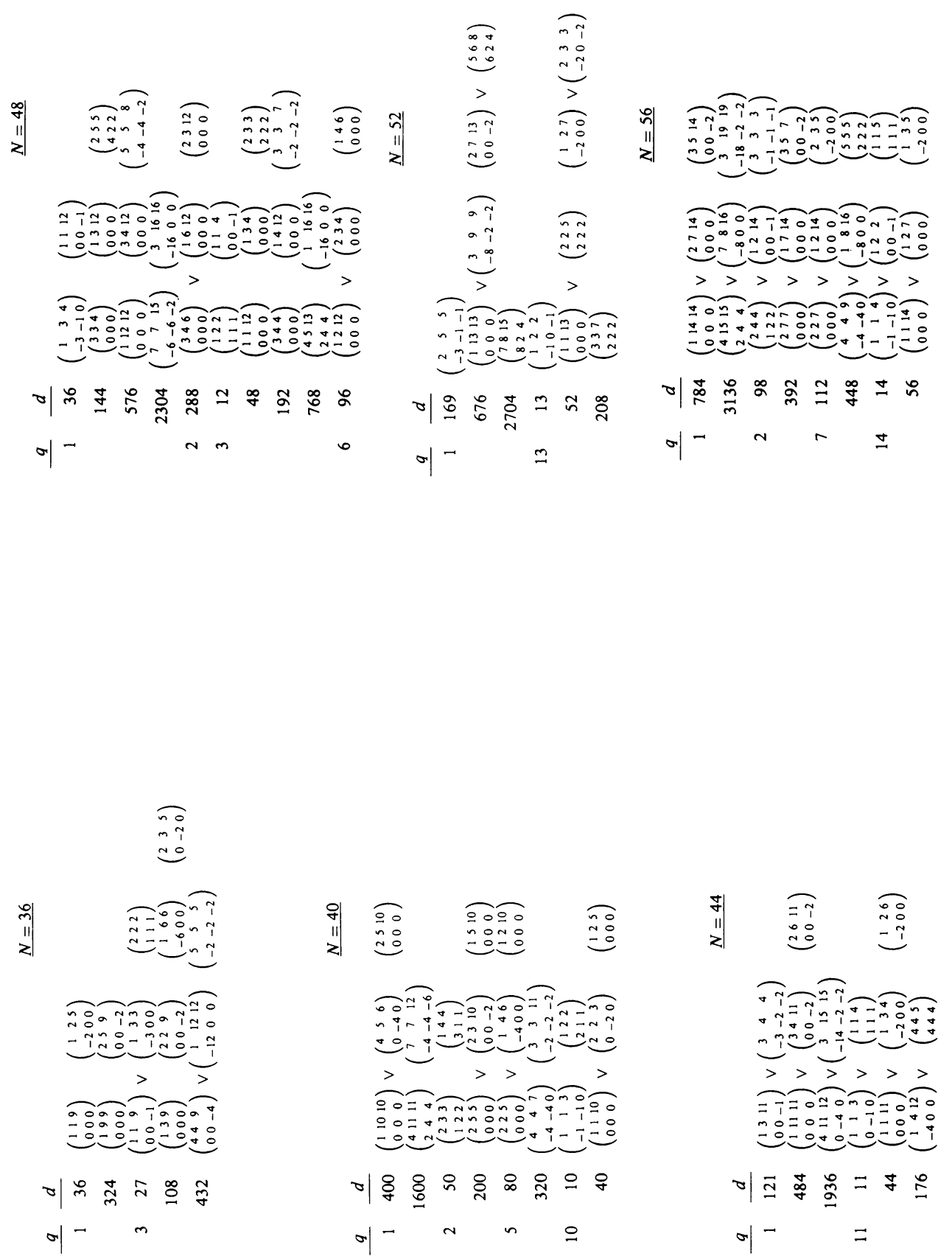


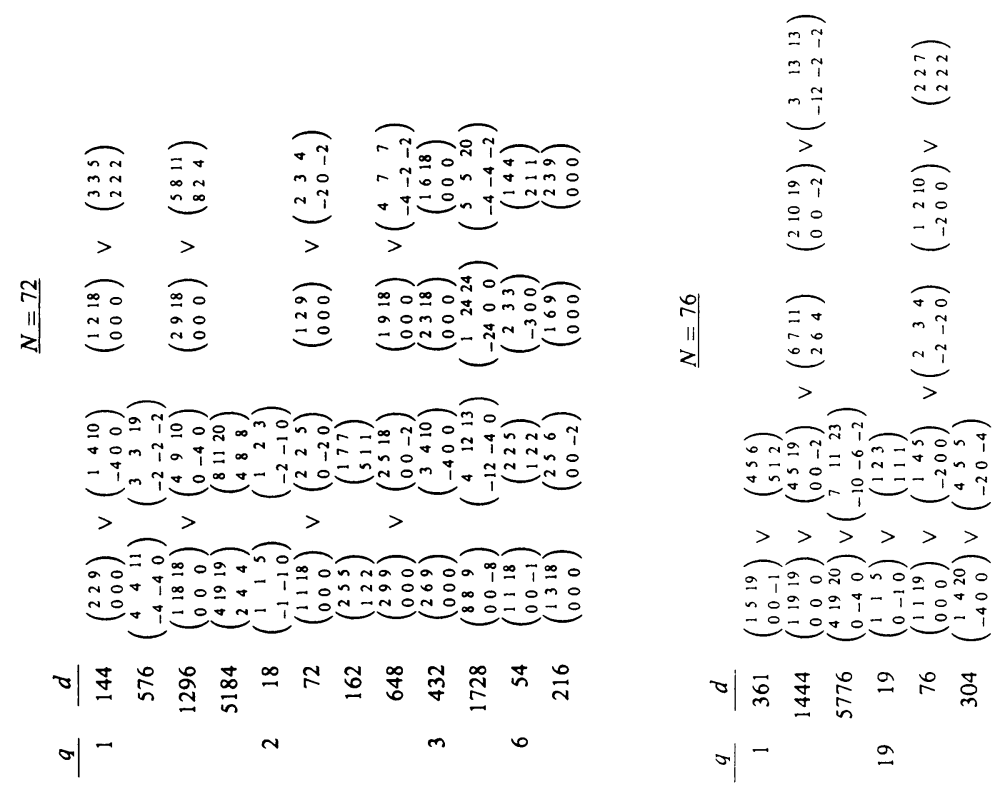

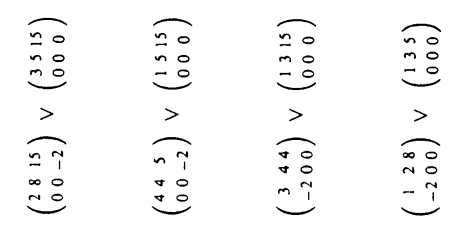

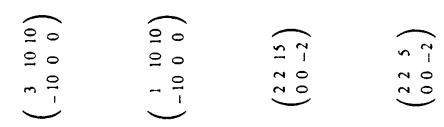

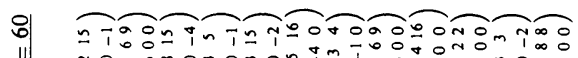

II)

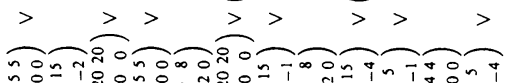

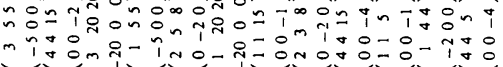

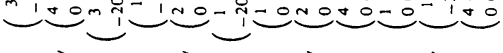

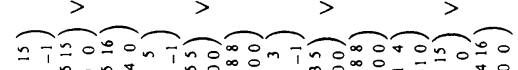

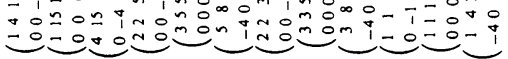

이 졍 \& or $\cong$

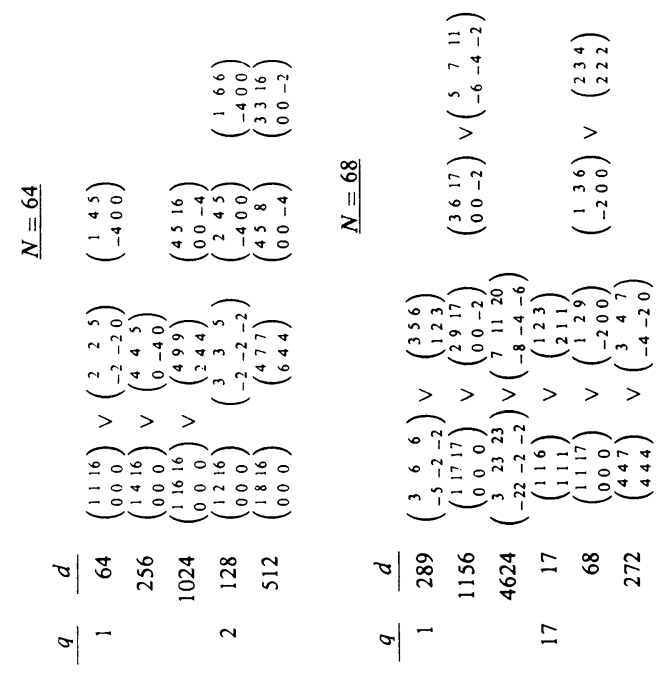




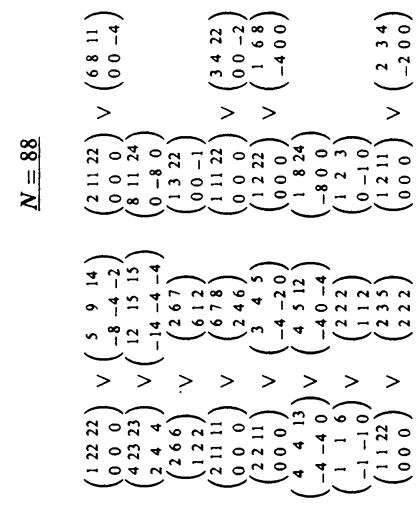

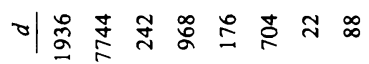

$$
\text { of } \quad=\pi
$$

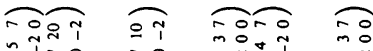

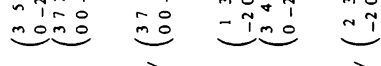

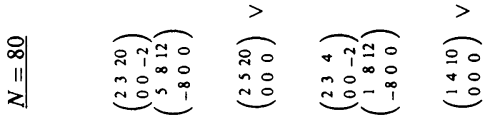

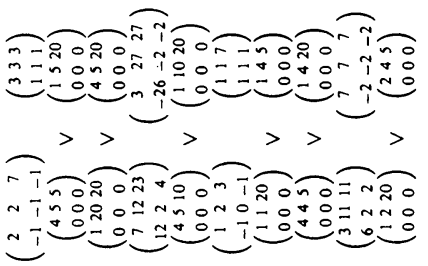

기의 \& \& \& \& \& of

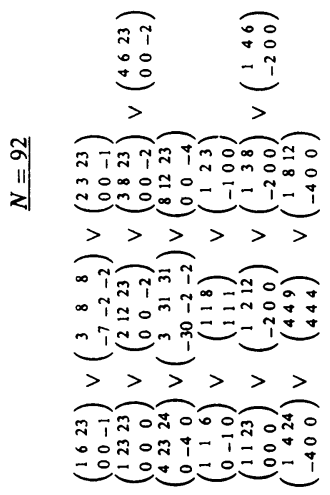

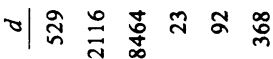

of

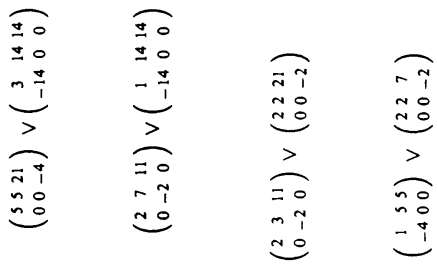

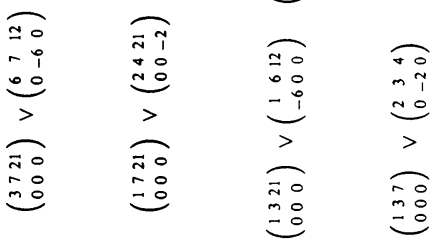

\section{म)}

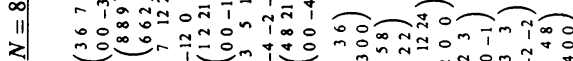

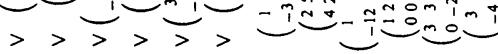

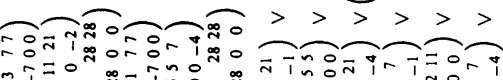

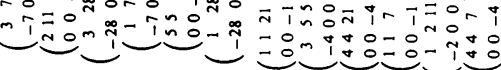

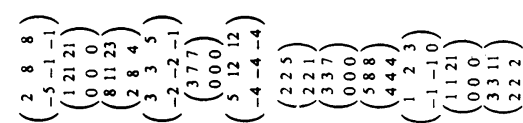

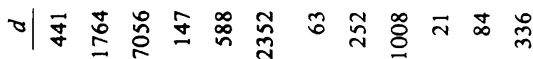

of $m \quad \vec{N}$ 


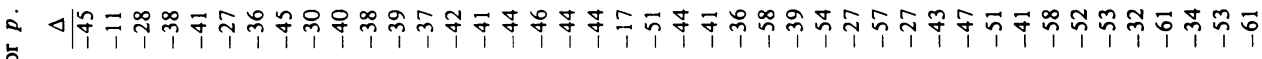

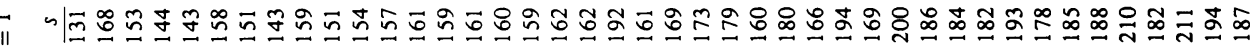

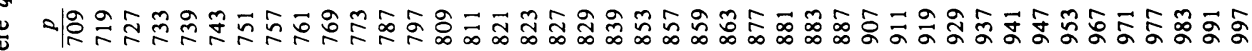
$\hat{x}$

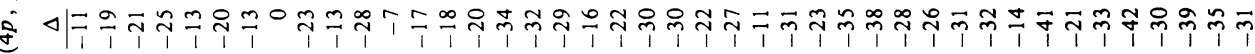
"

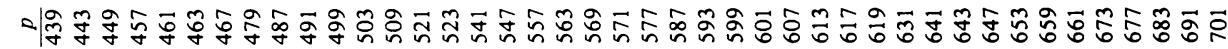

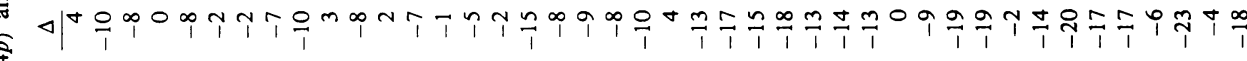

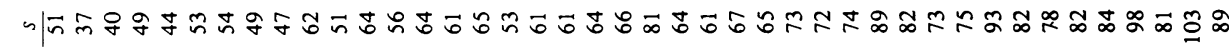

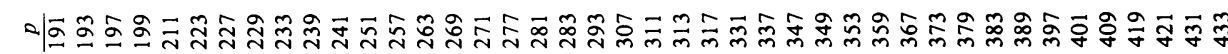
$\checkmark$ हू 曾

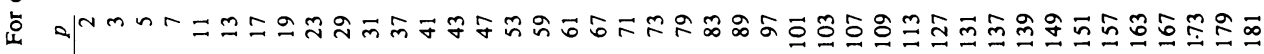
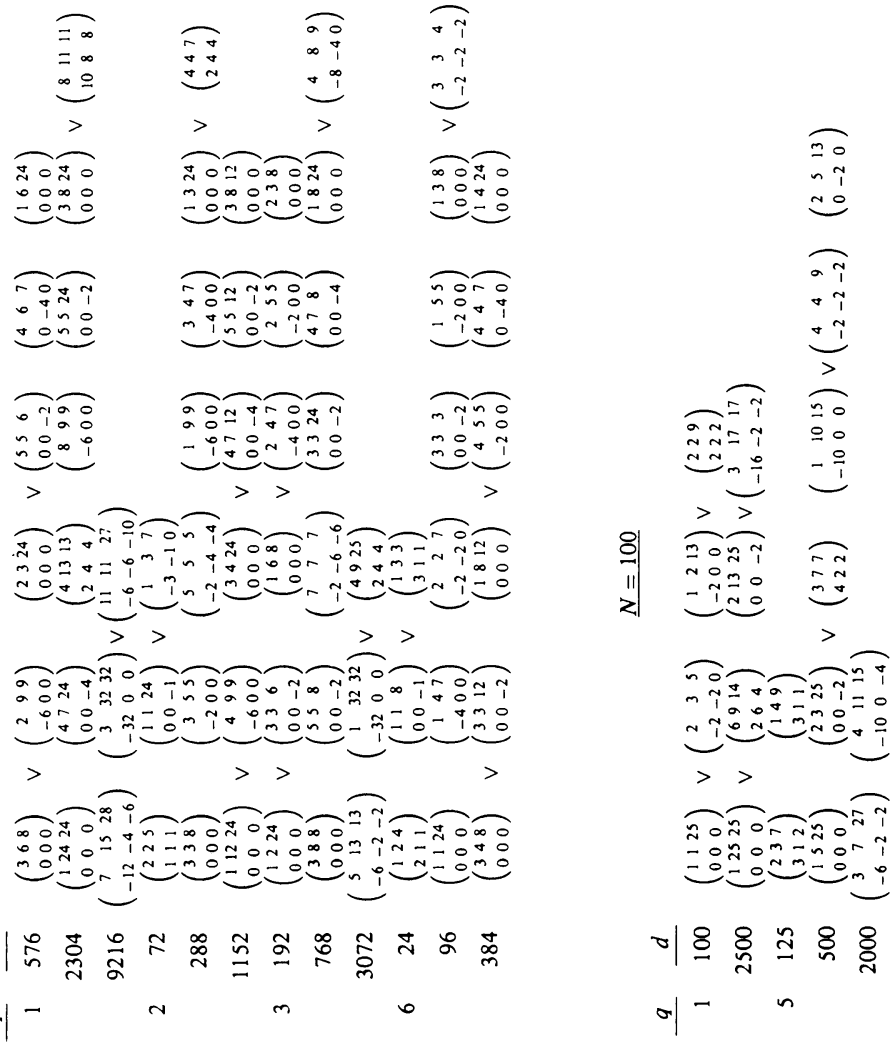

2

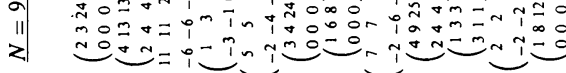

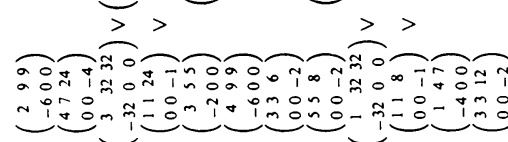

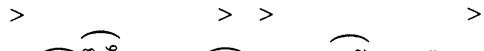

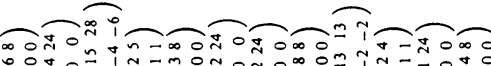
no.0.

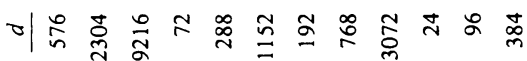
of m $\quad$ o 